\title{
LIMIT PERIODIC JACOBI MATRICES WITH A PRESCRIBED p-ADIC HULL AND A SINGULAR CONTINUOUS SPECTRUM
}

\author{
F. Peherstorfer, A. Volberg, P. Yuditski
}

\begin{abstract}
For all hyperbolic polynomials we proved in [9] a Lipschitz estimate of Jacobi matrices built by orthogonalizing polynomials with respect to measures in the orbit of classical Perron-Frobenius-Ruelle operators associated to hyperbolic polynomial dynamics (with real Julia set). Here we prove that for all sufficiently hyperbolic polynomials this estimate becomes exponentially better when the dimension of the Jacobi matrix grows. In fact, our main result asserts that a certain natural non-linear operator on Jacobi matrices built by a hyperbolic polynomial with real Julia set is a contraction in operator norm if the polynomial is sufficiently hyperbolic. This allows us to get for such polynomials the solution of a problem of Bellissard, in other words, to prove the limit periodicity of the limit Jacobi matrix. This fact does not require the iteration of the same fixed polynomial, and therefore it gives a wide class of limit periodic Jacobi matrices with singular continuous spectrum.
\end{abstract}

\section{Introduction}

Let $T$ be an expanding polynomial with the real Julia set $\operatorname{Julia}(T), \operatorname{deg} T=d$. We recall that Julia $(T)$ is a nonempty compact set of points which do not go to infinity under forward iterations of $T$. Under the normalization

$$
T^{-1}:[-1,1] \rightarrow[-1,1] ; \pm 1 \in \operatorname{Julia}(T)
$$

such a polynomial is well-defined by the position of its critical values

$$
C V(T):=\left\{t_{i}=T\left(c_{i}\right): T^{\prime}\left(c_{i}\right)=0, c_{i}>c_{j} \text { for } i>j\right\} .
$$

Expanding, or hyperbolic polynomials are those, for which

$$
c_{i} \notin \operatorname{Julia}(T), \forall i
$$

which is the same as to say that $C V(T) \cap \operatorname{Julia}(T)=\emptyset$ (just use the fact that Julia $(T)$ is invariant under taking full preimage $\left.T^{-1}\right)$. The term "expanding" is deserved because for expanding polynomials one has the following inequality

$$
\exists Q>1,\left|\left(T^{n}\right)^{\prime}(x)\right| \geq c Q^{n}, \forall x \in \operatorname{Julia}(T) .
$$

Received by the editors October 25, 2004. Revised October 3, 2005.

Partially supported by NSF grant DMS-0200713, the Austrian Science Fund FWF, project number: P16390-N04 and Marie Curie International Fellowship within the 6-th European Community Framework Programme, Contract Number: MIF1-CT-2005-006966.

AMS subject classification codes: 42B20, 42C15, 42A50, 47B35, 47B38.

Key words: almost periodic Jacobi matrices, singular continuous spectrum, hyperbolic polynomials, harmonic measure. 
Here and in everything what follows $T^{n}$ means $n$-th iteration of $T, T^{n}=T \circ T \circ$ ....T.

Let us mention that for $T$ with a real Julia set one has $\left|T\left(c_{i}\right)\right|>1$ since all solutions of $T(x)= \pm 1$ should be real.

We will need to consider the notion of "sufficiently expanding" ("sufficiently hyperbolic") polynomials. As we saw, the expanding property is the same (in our normalization) as $\operatorname{dist}(C V(T),[-1,1])>0$. The polynomial $T$ with normalization (1.1) will be called sufficiently hyperbolic (or sufficiently expanding) if

$$
\operatorname{dist}(C V(T),[-1,1]) \geq A,
$$

where $A$ is a large absolute constant to be specified later (but $A=10$ will work). Notice that the definition of sufficient hyperbolicity does not involve the degree of $T$. In particular, $T$ and any of its iterative powers $T^{2}, T^{3}, \ldots$ are sufficiently hyperbolic simultaneously.

A Jacobi matrix $J: l^{2}(\mathbb{Z}) \rightarrow l^{2}(\mathbb{Z})$ is called almost periodic if the family

$$
\left\{S^{-k} J S^{k}\right\}_{k \in \mathbb{Z}}
$$

where $S$ is the shift operator in $l^{2}(\mathbb{Z}), S|m\rangle=|m+1\rangle$, is a precompactum in the operator topology.

Example. Let $G$ be a compact abelian group, $p(\alpha), q(\alpha)$ be continuous functions on $G, p(\alpha) \geq 0$. Then $J(\alpha)$ with the coefficient sequences $\{p(\alpha+k \mu)\}_{k},\{q(\alpha+$ $k \mu)\}_{k}, \mu \in G$, is almost periodic.

Let us show that in fact this is a general form of almost periodic Jacobi matrices. For a given almost periodic $J$ define the metric on $\mathbb{Z}$ by

$$
\rho_{J}(k):=\left\|S^{-k} J S^{k}-J\right\| .
$$

Evidently $\rho_{J}(k+m) \leq \rho_{J}(k)+\rho_{J}(m)$. Then $J=J(0)$, where $G=I_{J}, I_{J}$ is the closure of $\mathbb{Z}$ with respect to $\rho_{J}$, and $\mu=1 \in I_{J}$.

Recall that for a given system of integers $\left\{d_{k}\right\}_{k=1}^{\infty}$ one can define the set

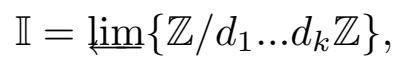

that is $\alpha \in \mathbb{I}$ means that $\alpha$ is a sequence $\left\{\alpha_{0}, \alpha_{1}, \alpha_{2}, \ldots\right\}$ such that

$$
\alpha_{k} \in \mathbb{Z} / d_{1} \ldots d_{k+1} \mathbb{Z} \quad \text { and } \quad \alpha_{k} \mid \bmod d_{1} \ldots d_{k}=\alpha_{k-1} .
$$

The addition in $\mathbb{I}$ is defined as addition in the $l$-th entry. The metric $\operatorname{dist}(\alpha, \beta)=$ $\kappa^{l}$, where $\kappa \in(0,1), l$ is the smallest integer such that $\alpha_{l} \neq \beta_{l}$, makes II a compact abelian group. In particular, if $p$ is a prime number and $d_{k}=p$ we get the ring of $p$-adic integers, $\mathbb{I}=\mathbb{Z}_{p}$.

In this work we build a certain machinery that allows to construct almost periodic Jacobi matrices with singularly continuous spectrum such that $I_{J}=\mathbb{I}$.

The key element of the construction is the following 
Theorem 1.1. Let $\tilde{J}$ be a Jacobi matrix with the spectrum on $[-1,1]$. Then the following Renormalization Equation has a solution $J=J(\tilde{J})=J(\tilde{J} ; T)$ with the spectrum on $T^{-1}([-1,1])$ :

$$
V^{*}(z-J)^{-1} V=(T(z)-\tilde{J})^{-1} T^{\prime}(z) / d,
$$

where $V|k\rangle=|d k\rangle$. Moreover, if $\min _{i}\left|t_{i}\right| \geq 10$ then

$$
\left\|J\left(\tilde{J}_{1}\right)-J\left(\tilde{J}_{2}\right)\right\| \leq \kappa|| \tilde{J}_{1}-\tilde{J}_{2} \| .
$$

with an absolute constant $\kappa<1$ (does not depend on $T$ also).

This theorem, for example, will result in the following consequence:

Theorem 1.2. Let $T$ be sufficiently hyperbolic in the sense of (1.3). Let $J_{\omega}$ be the Jacobi matrix obtained by orthogonalizing polynomials with respect to the balanced (equilibrium) measure $\omega$ on the Julia set of $T$. Then $J_{\omega}$ is a limit periodic matrix. In other words, the sequences that give the diagonal and the below (above) diagonal entries are uniform limits of periodic sequences.

Remarks. 1) A known problem (due to Bellissard) asks to prove this statement for all hyperbolic polynomials normalized as in (1.1). Here we do it for all sufficiently hyperbolic polynomials (recall that we always tacitly assume that $\mathrm{Julia}(T)$ is on the real line). Our result about sufficiently hyperbolic polynomials explains, in a sense, the earlier results in [2], [3], where it has been shown that polynomials $T(z):=\varepsilon^{-n} \mathcal{T}_{n}(\varepsilon z)$ generate limit periodic Jacobi matrices if $\varepsilon$ is sufficiently small, here $\mathcal{T}_{n}$ is the $n$-th Tchebyshef polynomial. Smallness of $\varepsilon$ obviously makes $T=\varepsilon^{-n} \mathcal{T}_{n}(\varepsilon z)$ sufficiently hyperbolic in our sense (1.3).

2) In the thesis of Herndon [6] Theorem 1.2 is proved by another method. We regret that it has not been published, that might have clarified the proof, which seems to be quite involved.

3) One can wonder after analyzing the results of [2], [3] and the present paper, that may be there is a threshold of hyperbolicity: before it $J_{\omega}$ is not limit periodic, and after it it is limit periodic. However, we do not believe in this sort of behavior, but at this stage we cannot prove the conjecture of Bellissard for all hyperbolic polynomial with real Julia set.

4) Let us mention that, in fact, (1.5) has $2^{d-1}$ solutions such that the spectrum of $J$ is on $T^{-1}([-1,1])$. Here we use only one of them.

We note that the real output of Theorem 1.1 is much wider than Theorem 1.2. It shows that

a) roughly speaking, constructing in a regular iterative way a Cantor set $E$, $E \subset \cdots \subset E_{n+1} \subset E_{n} \ldots$, that may support the spectrum of a limitperiodic Jacobi matrix it is enough to follow the strategy: on each step the approximating set $E_{n}$ should have a form of an inverse polynomial image, i.e.:

$$
E_{n}=U_{n}^{-1}[-1,1], \quad U_{n} \text { is a polynomial },
$$


or, what is the same, $E_{n}$ should be the spectrum of a periodic Jacobi matrix;

b) the above statement becomes a theorem if on each step we remove from the previous set a sufficiently large part (using sufficiently expanding polynomials), i.e.: if $T_{1}, T_{2} \ldots$, is a sequence of polynomials with sufficiently large critical values, then $E_{n}:=U_{n}^{-1}[-1,1]$, with $U_{n}=T_{n} \circ \ldots \circ T_{2} \circ T_{1}$;

c) the set $E$, that was constructed in the above described way, is the spectral set of infinitely many (uncountable set) of different limit periodic Jacobi matrices, that means that each of the matrices does not belong to the hull of another one (can't be obtained as a limit of shifts) [7], [10]. The problem: to describe the set of all limit periodic Jacobi matrices with spectrum $E$ or certain subclasses (or at least to try to understand how these sets look like), is a very interesting and challenging problem.

Let us outline a proof of claim b). First, we point out the following two properties of the function $J(\tilde{J} ; T)$ in Theorem 1.1. Due to the commutant relation $V S=S^{d} V$ one gets $J\left(S^{-m} \tilde{J} S^{m}\right)=S^{-d m} J(\tilde{J}) S^{d m}$. The second property is

$$
J\left(J\left(\tilde{J} ; T_{2}\right) ; T_{1}\right)=J\left(\tilde{J} ; T_{2} \circ T_{1}\right),
$$

that is, the chain rule holds.

Now, we produce a limit periodic Jacobi matrix with singularly continuous spectrum and such that $I_{J}=\mathbb{I}$. For the chosen system of polynomials $T_{1}, T_{2} \ldots$, $\operatorname{deg} T_{k}=d_{k}$, with sufficiently large critical values, define $J_{m}=J\left(\tilde{J} ; T_{m} \circ \ldots \circ T_{2} \circ\right.$ $T_{1}$ ). By Theorem 1.1, the limit $J=\lim _{m \rightarrow \infty} J_{m}$ exists and does not depend on $\tilde{J}$. Moreover,

$$
\forall j,\left\|J-S^{-d_{1} \ldots d_{l} j} J S^{d_{1} \ldots d_{l} j}\right\| \leq\left\|J-S^{-j} J S^{j}\right\| \kappa^{l} \leq 2 \kappa^{l} .
$$

That is $\rho_{J}$ defines on $\mathbb{Z}$ the standard $p$-adic topology in this case. This proves that $J$ is a limit periodic matrix, in particular, it is almost periodic.

Notice that for the case $T_{1}=T_{2}=\ldots=T_{m}=: T$, we get the limit periodic matrix with the spectrum on $\operatorname{Julia}(T)$.

For an extended version of the current paper see [10], where we consider (the most natural) action of the renormalization on periodic Jacobi matrices, describe other solutions of the Renormalization Equation and their relations with the Ruelle operators, prove the Lipschitz property of the Darboux transform via our main theorem, etc.

\section{Renormalization equation}

In this section it is convenient to assume that

$$
T(z)=z^{d}-q d z^{d-1}+\ldots
$$

is a monic expanding polynomial. Under this normalization $T^{-1}:[-\xi, \xi] \rightarrow$ $[-\xi, \xi]$ for a certain $\xi>0$. 
Let $\tilde{J}: l^{2}(\mathbb{Z}) \rightarrow l^{2}(\mathbb{Z})$ be a Jacobi matrix with the spectrum on $[-\xi, \xi]$. We describe the set of solutions of the Renormalization Equation

$$
V^{*}(z-J)^{-1} V=(T(z)-\tilde{J})^{-1} T^{\prime}(z) / d, \quad V|k\rangle=|k d\rangle,
$$

here $J$ is a Jacobi matrix with the spectrum on $T^{-1}([-\xi, \xi])$ that should satisfy (2.1).

In what follows by $l_{ \pm}^{2}(s)$ we denote the spaces which are formed by $\{\mid s+$ $k\rangle$ \} with $k \leq 0$ and $k \geq 0$ respectively, that is $l^{2}(\mathbb{Z})=l_{-}^{2}(s) \oplus l_{+}^{2}(s+1)$. Correspondingly to these decompositions we set $\tilde{J}_{ \pm}(s)=P_{l_{ \pm}^{2}(s)} \tilde{J} \mid l_{ \pm}^{2}(s)$.

Recall that a (finite or infinite) one-sided Jacobi matrix is uniquely determined by its so called resolvent function

$$
\tilde{r}_{ \pm}(z, s)=\left\langle s\left|\left(\tilde{J}_{ \pm}(s)-z\right)^{-1}\right| s\right\rangle,
$$

for which the following decomposition in the continued fraction holds true

$$
\tilde{r}_{+}(z, s)=\frac{-1}{z-\tilde{q}_{s}-\frac{\tilde{p}_{s+1}^{2}}{z-\tilde{q}_{s+1}-\ldots}}=\frac{-1}{z-\tilde{q}_{s}+\tilde{p}_{s+1}^{2} \tilde{r}_{+}(z, s+1)} .
$$

Lemma 2.1. Assume that a matrix $J$ satisfies (2.1). Then

$$
p_{s d+1} \ldots p_{s d+d}=\tilde{p}_{s+1} \quad \text { and } \quad q_{s d}=q .
$$

Further, let $J^{(s)}$ be the $s$-th $d \times d$ block of $J$, that is,

$$
J^{(s)}=\left[\begin{array}{ccccc}
q_{s d} & p_{s d+1} & & & \\
p_{s d+1} & q_{s d+1} & p_{s d+2} & & \\
& \ddots & \ddots & \ddots & \\
& & p_{s d+d-2} & q_{s d+d-2} & p_{s d+d-1} \\
& & & p_{s d+d-1} & q_{s d+d-1}
\end{array}\right] .
$$

Then its resolvent function is of the form

$$
\left\langle 0\left|\left(z-J^{(s)}\right)^{-1}\right| 0\right\rangle=\frac{T^{\prime}(z) / d}{T^{(s)}(z)},
$$

where $T^{(s)}(z)$ is a monic polynomial of degree $d$. Moreover, at the critical points $\left\{c: T^{\prime}(c)=0\right\}$ the following recurrence relation holds

$$
T^{(s)}(c)+\frac{\tilde{p}_{s}^{2}}{T^{(s-1)}(c)}=T(c)-\tilde{q}_{s} .
$$

Proof. We write the Jacobi matrix $J$ as a $d \times d$ block matrix (each block is of infinite size), that is, we are just reordering the standard basis:

$$
J=\left[\begin{array}{ccccc}
\mathcal{Q}_{0} & \mathcal{P}_{1} & & & S \mathcal{P}_{d} \\
\mathcal{P}_{1} & \mathcal{Q}_{1} & \mathcal{P}_{2} & & \\
& \ddots & \ddots & \ddots & \\
& & \mathcal{P}_{d-2} & \mathcal{Q}_{d-2} & \mathcal{P}_{d-1} \\
\mathcal{P}_{d} S^{*} & & & \mathcal{P}_{d-1} & \mathcal{Q}_{d-1}
\end{array}\right] .
$$


Here $\mathcal{P}_{k}$ (respectively $\mathcal{Q}_{k}$ ) is a diagonal matrix $\mathcal{P}_{k}=\operatorname{diag}\left\{p_{k+s d}\right\}_{s \geq 0}$ and $S$ is the shift operator. With respect to this reordering $V^{*}$ is the projection on the first-place block-component.

Using this representation and the well known identity for block matrices

$$
\left[\begin{array}{ll}
A & B \\
C & D
\end{array}\right]^{-1}=\left[\begin{array}{cc}
\left(A-B D^{-1} C\right)^{-1} & * \\
* & *
\end{array}\right],
$$

we get

$$
\frac{T(z)-\tilde{J}}{T^{\prime}(z) / d}=z-\mathcal{Q}_{0}-\left[\mathcal{P}_{1}, \quad \ldots, \quad S \mathcal{P}_{d}\right]\left\{z-J_{1}\right\}^{-1}\left[\begin{array}{c}
\mathcal{P}_{1} \\
\vdots \\
\mathcal{P}_{d} S^{*}
\end{array}\right]
$$

where $J_{1}$ is the matrix that we obtain from $J$ by deleting the first block-row and the first block-column in (2.8). Thus the second relation in (4.1) is already proved, we just compare the leading terms in the decomposition over powers of $1 / z$ in the right and left hand sides and note that the third term on the right is of order $1 / z$.

But the most important remark is that in $\left(z-J_{1}\right)$ each block is a diagonal matrix (means all diagonals are the main diagonals in each block, on the contrary to $J$ that contains $S \mathcal{P}_{d}$ and $\mathcal{P}_{d} S^{*}$ ). That's why we can easily get an inverse matrix in terms of the scalar orthogonal polynomials.

Let us introduce the following notations: everything related to $J^{(s)}$ has superscript $s$. For instance: $p_{k}^{(s)}=p_{s d+k}, 1 \leq k \leq d$, respectively $P_{d}^{(s)}$ and $Q_{d}^{(s)}$ mean orthonormal polynomials of the first and second kind, in particular,

$$
\left\langle 0\left|\left(z-J^{(s)}\right)^{-1}\right| 0\right\rangle=\frac{Q_{d}^{(s)}(z)}{P_{d}^{(s)}(z)} .
$$

Let $J_{1}^{(s)}$ denote the matrix that we obtain from $J^{(s)}$ (see $(2.5)$ ) by deleting the first row and the first column. Then, for $J_{1}^{(s)}, Q_{d}^{(s)}$ are orthogonal polynomials of the first kind and we denote by $R_{d}^{(s)}$ corresponding to them orthogonal polynomials of the second kind. Note that $P_{d}^{(s)}$ and $R_{d}^{(s)}$ are related by

$$
\frac{P_{d}^{(s)}(z)}{Q_{d}^{(s)}(z)}=z-q_{0}^{(s)}-\left(p_{1}^{(s)}\right)^{2} \frac{R_{d}^{(s)}(z)}{Q_{d}^{(s)}(z)} .
$$

In these terms the four interesting for us elements of the resolvent of $J_{1}^{(s)}$ are:

$$
\left(z-J_{1}^{(s)}\right)^{-1}=\left[\begin{array}{ccc}
\frac{R_{d}^{(s)}}{Q_{d}^{(s)}} & \cdots & \frac{1}{p_{1}^{(s)} p_{d}^{(s)} Q_{d}^{(s)}} \\
\vdots & & \vdots \\
\frac{1}{p_{1}^{(s)} p_{d}^{(s)} Q_{d}^{(s)}} & \cdots & \frac{Q_{d-1}^{(s)} / p_{d}^{(s)}}{Q_{d}^{(s)}}
\end{array}\right] .
$$


Recalling again that $J_{1}$ is just a block-diagonal matrix we substitute (2.12) in (2.9). As result in the RHS (as well as in the LHS) we get a three-diagonal matrix. On the main diagonal we have

$$
z-\mathcal{Q}_{0}-\mathcal{P}_{1}\left\{\left(z-J_{1}\right)^{-1}\right\}_{1,1} \mathcal{P}_{1}-S \mathcal{P}_{d}\left\{\left(z-J_{1}\right)^{-1}\right\}_{d-1, d-1} \mathcal{P}_{d} S^{*}
$$

and each entry on the diagonal, due to (2.12) and then (2.11), is

$$
\begin{aligned}
z-q_{0}^{(s+1)}-\left(p_{1}^{(s+1)}\right)^{2} & \frac{R_{d}^{(s+1)}(z)}{Q_{d}^{(s+1)}(z)}-\left(p_{d}^{(s)}\right)^{2} \frac{Q_{d-1}^{(s)}(z) / p_{d}^{(s)}}{Q_{d}^{(s)}(z)} \\
= & \frac{P_{d}^{(s+1)}(z)}{Q_{d}^{(s+1)}(z)}-\left(p_{d}^{(s)}\right)^{2} \frac{Q_{d-1}^{(s)}(z) / p_{d s}^{(s)}}{Q_{d}^{(s)}(z)}
\end{aligned}
$$

Comparing this with the LHS (2.9) we get

$$
\frac{T(z)-\tilde{q}_{s+1}}{T^{\prime}(z) / d}=\frac{P_{d}^{(s+1)}(z)}{Q_{d}^{(s+1)}(z)}-\left(p_{d}^{(s)}\right)^{2} \frac{Q_{d-1}^{(s)}(z) / p_{d}^{(s)}}{Q_{d}^{(s)}(z)} .
$$

Similarly, below the main diagonal on the right we have

$$
-S \mathcal{P}_{d}\left\{\left(z-J_{1}\right)^{-1}\right\}_{d-1,1} \mathcal{P}_{1}
$$

So, using (2.12), we get from (2.9)

$$
\frac{\tilde{p}_{s+1}}{T^{\prime}(z) / d}=\frac{1}{Q_{d}^{(s)}(z)}=\frac{p_{1}^{(s)} \ldots p_{d}^{(s)}}{z^{d-1}+\ldots} .
$$

Thus the first relation in (4.1) is also proved, moreover all $Q_{d}^{(s)}(z)$ (independently on $s$ ), being normalized to the leading coefficient one, coincides with $T^{\prime}(z) / d$.

We define $T^{(s)}(z)=z^{d}+\ldots$ by the same normalization

$$
T^{(s)}(z):=\tilde{p}_{s+1} P_{d}^{(s)}(z) .
$$

Then (2.10) implies (2.6).

Now we use the (last) well known fact on orthogonal polynomials - the Wronskian identity:

$$
p_{d}^{(s)} Q_{d}^{(s)}(z) P_{d-1}^{(s)}(z)-p_{d}^{(s)} P_{d}^{(s)}(z) Q_{d-1}^{(s)}(z)=1,
$$

due to which, if $T^{\prime}(c)=0$ then

$$
-p_{d}^{(s)} Q_{d-1}^{(s)}(c)=\frac{1}{P_{d}^{(s)}(c)} .
$$

So, combining (2.16) with (2.14), we get from (2.13) the recurrence relation

$$
T(c)-\tilde{q}_{s+1}=T^{(s+1)}(c)+\frac{\tilde{p}_{s+1}^{2}}{T^{(s)}(c)} .
$$

Thus the lemma is completely proved. 
Now we are in a position to show that the Renormalization Equation has $2^{d-1}$ solutions. Then we show that they are the only possible solutions. This set of solutions we parametrize by a collections of vectors

$$
\delta:=\left\{\delta_{c}\right\}_{c},
$$

where each component $\delta_{c}$ can be chosen as plus or minus one.

Theorem 2.2. Fix a vector $\delta$ of the form (2.18). For a given $\tilde{J}$ with the spectrum on $[-\xi, \xi]$ define the Jacobi matrix $J$ according to the following algorithm:

For $s \in \mathbb{Z}$ we put

$$
\frac{1}{T^{(s)}(c)}=-\tilde{r}_{-}(T(c), s), \quad \text { if } \delta_{c}=-1
$$

and

$$
T^{(s)}(c)=-\tilde{p}_{s+1}^{2} \tilde{r}_{+}(T(c), s+1), \quad \text { if } \delta_{c}=1
$$

where the functions $\tilde{r}_{ \pm}(z, s)$ are defined by (2.2). Then define the monic polynomial $T^{(s)}(z)$ of degree $d$ by the interpolation formula

$$
T^{(s)}(z)=(z-q) T^{\prime}(z) / d+\sum_{c: T^{\prime}(c)=0} \frac{T^{\prime}(z)}{(z-c) T^{\prime \prime}(c)} T^{(s)}(c) .
$$

Define the block $J^{(s)}$ (see (2.5)) by its resolvent function according to (2.6). Finally define the entry $p_{s d+d}$ by $(4.1)$.

We claim that the matrix $J=J(\delta, \tilde{J})$, combined with such blocks and entries over all s, satisfies (2.1).

Example. The solution related to the vector

$$
\delta_{-}=\{-1, \ldots,-1\}
$$

that is all $T^{(s)}(c)$ are defined by $(2.19)$, plays the most important role in what follows. Precisely for this solution $J(\tilde{J}):=J\left(\delta_{-}, \tilde{J}\right)$ we prove the contractibility property (our main Theorem 1.1).

Proof. First of all let us mention that for all $c,|T(c)|>\xi$, that is $T^{(s)}(c)$ is well defined by either (2.19) or (2.20), moreover this value is of the same sign as $T(c)$. It means that the rational function $-\frac{T^{\prime}(z) / d}{T^{(s)}(z)}$ is the Stieltjes transform of a positive measure (supported at the zeros of $T^{(s)}(z)$ ), and hence there exists a unique $d \times d$ Jacobi matrix defined by (2.5). Note that (4.2) implies immediately that $q_{s d}=q$, again we look at the leading term in the decomposition of the resolvent function into the continued fraction.

Now, we see that $(2.14)$ holds, it's just a matter of the definition of $p_{d}^{(s)}$. We have to check (2.13). Using the form of $Q_{d}^{(s)}(z)$ we have equivalently

$$
T(z)-\tilde{q}_{s+1}=T^{(s)}(z)+\tilde{p}_{s+1} p_{d}^{(s)} Q_{d-1}^{(s)}(z) .
$$

Subtracting $(z-q) T^{\prime}(z) / d$ from both parts we arrive at the question of the identity of two polynomials of degree $d-2$. Thus, we need to check (2.22) only at 
the critical points. Using the Wronskian identity (2.16) we get (2.17). Of course, the main point is that $T^{(s)}(c)$, being defined by either (2.19) or (2.20), satisfy the recursion (2.7) (this is the continued fraction decomposition for $\tilde{r}_{ \pm}(z, s)$, see $(2.3))$.

Thus having (2.9), we proved (2.1).

Theorem 2.3. Theorem 2.2 describes the whole set of solutions of the Renormalization Equation.

Proof. We need to show that (2.19), (2.20) give the complete possible choice of the values $T^{(s)}(c)$, say for $s=0$. Then all other values are of the same form due to Lemma 2.1.

We claim that any other choice of $T^{(0)}(c)$ contradicts to the regularity of the resolvent of $J$ at $c$.

Using standard formulas for orthogonal polynomials for two-sided Jacobi matrices (see Appendix, Corollary 5.2) we have from the Renormalization Equation

$$
\begin{gathered}
\tilde{r}_{-}^{-1}(T(z), 0)=r_{-}^{-1}(z, 0) T^{\prime}(z) / d+p_{1}^{2} \tilde{p}_{1} R_{d}^{(0)}(z), \\
\tilde{p}_{1}^{2} \tilde{r}_{+}(T(z), 1)=p_{1}^{2} r_{+}(z, 1) T^{\prime}(z) / d+p_{1}^{2} \tilde{p}_{1} R_{d}^{(0)}(z),
\end{gathered}
$$

where $r_{ \pm}(z, s)$ are the resolvent functions of $J_{ \pm}(s)$.

In the same time both functions $r_{+}(z, 1)$ and $r_{-}^{-1}(z, 0)$ can not have a pole at $c$ simultaneously. This contradicts to (see (5.5))

$$
\left\langle 1\left|(J-z)^{-1}\right| 1\right\rangle=r_{+}(z, 1) r_{-}^{-1}(z, 0)\left\langle 0\left|(J-z)^{-1}\right| 0\right\rangle:
$$

$\left\langle 0\left|(J-z)^{-1}\right| 0\right\rangle$ can not have zero multiplicity more than one and $\left\langle 1\left|(J-z)^{-1}\right| 1\right\rangle$ should be regular at $c$.

By $(2.11) T^{(0)}(c)=-p_{1}^{2} \tilde{p}_{1} R_{d}^{(0)}(c)$, so we get $(2.19)$ from $(2.23)$ if $r_{-}^{-1}(z, 0)$ is regular and (2.20) in the case when $r_{+}(z, 1)$ is regular at $c$.

Remark. Formulas (2.19), (2.20) play the main role in solving the Renormalization Equation. Actually, we proved or found them in Theorem 2.3 for $s=0$, or, in the same way, for any other fixed $s$. However, there is another important ingredient: it should be also shown that if we choose this or that definition of $T^{(s)}(c)$ for any particular $s$ we have to be consistent with this definition for all other values of $s$, that is we can not define, for instance, $T^{(0)}(c)$ by $(2.19)$ and $T^{(1)}(c)$ by $(2.20)$ for the same $c$. Precisely this claim is the main output of Lemma 2.1.

Let us mention that the Renormalization Equation can be rewritten equivalently in the form of polynomials equations.

Lemma 2.4. Equation (2.1) is equivalent to

$$
\begin{gathered}
V^{*} T(J)=\tilde{J} V^{*} \\
V^{*} \frac{T(z)-T(J)}{z-J} V=T^{\prime}(z) / d .
\end{gathered}
$$


Proof. Starting with (2.25), (2.26) we get

$$
(T(z)-\tilde{J}) V^{*}(z-J)^{-1} V=V^{*}\{T(z)-T(J)\}\{z-J\}^{-1} V=T^{\prime}(z) / d .
$$

Having (2.1) we get

$$
\begin{aligned}
V^{*} \frac{T(z)-T(J)}{z-J} V & =T(z) V^{*}(z-J)^{-1} V-V^{*} \frac{T(J)}{z-J} V \\
& =T(z) \frac{T^{\prime}(z) / d}{T(z)-\tilde{J}}-V^{*} \frac{T(J)}{z-J} V \\
& =T^{\prime}(z) / d+\tilde{J} V^{*}(z-J)^{-1} V-V^{*} \frac{T(J)}{z-J} V .
\end{aligned}
$$

Since the left hand side in (2.27) is a polynomial of $z$ we obtain two relations

$$
V^{*} \frac{T(z)-T(J)}{z-J} V=T^{\prime}(z) / d
$$

and

$$
\left\{\tilde{J} V^{*}-V^{*} T(J)\right\}\left\{(z-J)^{-1} V\right\}=0 .
$$

Since vectors of the form $(z-J)^{-1} V f, f \in l^{2}$, are complete in $l^{2}$ the last relation implies (2.26).

\section{Proof of the main theorem}

We start with

Lemma 3.1. Let $J^{(s)}$ be the $s$-the block of $J=J(\delta, \tilde{J})$. Define the measure $\sigma^{(s)}$ by

$$
\frac{T^{(s)}(z)}{T^{\prime}(z) / d}=z-q-\int \frac{d \sigma^{(s)}(x)}{x-z},
$$

that is, $\sigma^{(s)}$ is the spectral measure of the obliterated matrix $J_{1}^{(s)}$ normalized by

$$
\int d \sigma^{(s)}(x)=\left(p_{1}^{(s)}\right)^{2}
$$

Then

$$
\int \frac{d \sigma^{(s)}}{T^{(s)}(c)^{2}}=\frac{p_{(s+1) d}^{2}}{\tilde{p}_{s+1}^{2}} .
$$

Proof. Note that $Q_{d-1}^{(s)}$ is the orthonormal polynomial with respect to $\sigma^{(s)}$,

$$
\int\left(Q_{d-1}^{(s)}(c)\right)^{2} d \sigma^{(s)}(c)=1
$$

Using (2.16) and the normalization (2.15) we get (3.2).

The proof of the theorem is based on the following well-known and simple lemma. 
Lemma 3.2. Assume that two non-normalized measures $\sigma$ and $\hat{\sigma}$ are mutually absolutely continuous. Moreover, that $d \hat{\sigma}=f d \sigma$ and $(1+\epsilon)^{-1} \leq f \leq(1+\epsilon)$. Let us associate with these measures Jacobi matrices $J=J(\sigma), \hat{J}=J(\hat{\sigma})$. Then for their coefficients we have

$$
\left|\hat{p}_{s}-p_{s}\right| \leq \epsilon|| J||, \quad s \geq 0 .
$$

Proof. Assume that $p_{s} \geq \hat{p}_{s}$. Let us use an extremal property of orthogonal polynomials,

$$
\begin{aligned}
(1+\epsilon) \hat{p}_{0}^{2} \ldots \hat{p}_{s}^{2} & =(1+\epsilon) \int \hat{p}_{0}^{2} \ldots \hat{p}_{s}^{2} \hat{P}_{s}^{2} d \hat{\sigma} \geq \int\left\{z^{s}+\ldots\right\}^{2} d \sigma \\
& \geq \inf _{\left\{P=z^{s}+\ldots\right\}} \int P^{2} d \sigma=p_{0}^{2} \ldots p_{s}^{2} .
\end{aligned}
$$

Similarly

$$
(1+\epsilon) p_{0}^{2} \ldots p_{s-1}^{2} \geq \hat{p}_{0}^{2} \ldots \hat{p}_{s-1}^{2}
$$

Therefore

$$
p_{s}^{2} \leq \hat{p}_{s}^{2} \leq(1+\epsilon)^{2} p_{s}^{2}
$$

and hence

$$
0 \leq \hat{p}_{s}-p_{s} \leq \epsilon p_{s}
$$

Proof of Theorem 1.1. Given $\tilde{J}_{I}$ and $\tilde{J}_{I I}$ let us compare the blocks $J_{I}^{(s)}$ and $J_{I I}^{(s)}$ of the matrices $J_{I}:=J\left(\delta_{-}, \tilde{J}_{I}\right)$ and $J_{I I}:=J\left(\delta_{-}, \tilde{J}_{I I}\right)$. Actually we will apply Lemma 3.2 to non-normalized spectral measures $\sigma_{I}^{(s)}$ and $\sigma_{I I}^{(s)}$, see (3.1), corresponding to the obliterated matrices $\left(J_{I}^{(s)}\right)_{1}$ and $\left(J_{I I}^{(s)}\right)_{1}$.

Note that both measures are supported on the critical points $\left\{c: T^{\prime}(c)=0\right\}$ and, therefore, they are mutually absolutely continuous. Moreover, the density of the second measure with respect to the first one is of the form

$$
f(c)=f^{(s)}(c):=\frac{T_{I I}^{(s)}(c)}{T_{I}^{(s)}(c)} .
$$

Assuming $f(c) \geq 1$ let us estimate $f(c)-1$ from above.

$$
\begin{aligned}
f(c)-1 & =\frac{1 / T_{I}^{(s)}(c)-1 / T_{I I}^{(s)}(c)}{1 / T_{I I}^{(s)}(c)} \\
& =\frac{\left\langle s\left|\left(T(c)-\tilde{J}_{I I,-}(s)\right)^{-1}\left(\tilde{J}_{I,-}(s)-\tilde{J}_{I I,-}(s)\right)\left(T(c)-\tilde{J}_{I,-}(s)\right)^{-1}\right| s\right\rangle}{1 / T_{I I}^{(s)}(c)} .
\end{aligned}
$$

Since the spectrum of $\tilde{J}_{I I,-}(s)$ is on $[-\xi, \xi]$ we get, by definition $(2.19)$,

$$
\left|\frac{1}{T_{I I}^{(s)}(c)}\right| \geq \frac{1}{|T(c)|+\xi},
$$


and, for the same reason,

$$
\|\left(T(c)-\tilde{J}_{i,-}\right)^{-1}|s\rangle \| \leq \frac{1}{|T(c)|-\xi}, i=I \text { or } i=I I .
$$

Therefore

$$
0 \leq f(c)-1 \leq\left\|\tilde{J}_{I}-\tilde{J}_{I I}\right\| \frac{|T(c)|+\xi}{(|T(c)|-\xi)^{2}}
$$

Thus, by Lemma 3.2, we obtain

$$
\left|\left(p_{I}\right)_{s d+k}-\left(p_{I I}\right)_{s d+k}\right| \leq \varkappa|| \tilde{J}_{I}-\tilde{J}_{I I}||, \quad 1 \leq k \leq d-1,
$$

where

$$
\varkappa:=\max _{c} \frac{|T(c)| / \xi+1}{(|T(c)| / \xi-1)^{2}} .
$$
3.1

$$
\frac{1}{\left(p_{i}\right)_{s d+1 \ldots\left(p_{i}\right)_{s d+d-1}}}=\frac{\left(p_{i}\right)_{(s+1) d}}{\tilde{p}_{s+1}} \leq \frac{1}{|T(c)| / \xi-1}, i=I \text { or } i=I I .
$$

Now,

$$
\begin{aligned}
\left(p_{I}\right)_{s d+d}-\left(p_{I I}\right)_{s d+d} & =\frac{\left(\tilde{p}_{I}\right)_{s+1}}{\left(p_{I}\right)_{s d+1} \ldots\left(p_{I}\right)_{s d+d-1}}-\frac{\left(\tilde{p}_{I I}\right)_{s+1}}{\left(p_{I I}\right)_{s d+1} \ldots\left(p_{I I}\right)_{s d+d-1}} \\
& =\frac{\left(\tilde{p}_{I}\right)_{s+1}-\left(\tilde{p}_{I I}\right)_{s+1}}{\left(p_{I}\right)_{s d+1} \ldots\left(p_{I}\right)_{s d+d-1}} \\
& +\frac{\left(\tilde{p}_{I I}\right)_{s+1}}{\left(p_{I}\right)_{s d+1} \ldots\left(p_{I}\right)_{s d+d-1}}\left(1-\frac{\left(p_{I}\right)_{s d+1} \ldots\left(p_{I}\right)_{s d+d-1}}{\left(p_{I I}\right)_{s d+1} \ldots\left(p_{I I}\right)_{s d+d-1}}\right)
\end{aligned}
$$

Using (3.6), (3.4) and Lemma 3.2 we obtain

$$
\begin{aligned}
\left|\left(p_{I}\right)_{s d+d}-\left(p_{I I}\right)_{s d+d}\right| & \leq\left\|\tilde{J}_{1}-\tilde{J}_{2}\right\| \max _{c} \frac{1}{|T(c)| / \xi-1} \\
& +\max _{c} \frac{1}{|T(c)| / \xi-1}\left\|\tilde{J}_{1}-\tilde{J}_{2}\right\| \varkappa / 2 \\
& =\left\|\tilde{J}_{1}-\tilde{J}_{2}\right\| \max _{c} \frac{1}{|T(c)| / \xi-1}(1+\varkappa / 2) .
\end{aligned}
$$

Thus (3.5) and (3.7) show that say for $\min _{c}|T(c)| / \xi \geq 10$ the renormalization is a contraction.

\section{Proof of Theorem 1.2}

Proof. For a given sufficiently hyperbolic polynomial $T$ we define

$$
J_{n+1}=J\left(\delta_{-}, J_{n}\right)
$$


starting from an arbitrary initial $J_{0}=\tilde{J}$ with the spectrum on $[-\xi, \xi]$. Due to the contractibility of the renormalization $J_{n}$ converges in the operator norm to $J$, moreover $J$ has the spectrum on $\operatorname{Julia}(T)$ and it is limit periodic

$$
\left\|J-S^{-d^{n} l} J S^{d^{n} l}\right\| \leq 2 \xi \kappa^{n}, \kappa<1 .
$$

We claim that $J$ is an orthogonal sum of two one-sided Jacobi matrices

$$
J=\left[\begin{array}{cc}
J_{-}(-1) & 0 \\
0 & J_{+}(0)
\end{array}\right]
$$

That is, we claim that $p_{0}=0$. Indeed, by Lemma 3.1 we have

$$
p(n+1)_{s d} \leq\left\{\max _{c} \frac{1}{|T(c)| / \xi-1}\right\} p(n)_{s} \leq \kappa p(n)_{s}
$$

where $p(n)_{s}$ is the $s$-th coefficient in the matrix $J_{n}$. Therefore, all $s d^{l}$-th coefficients of $J$ are uniformly small

$$
p_{s d^{l}} \leq 2 \xi \kappa^{l}
$$

and, in particular, $p_{0}=0$.

Thus $J_{+}:=J_{+}(0)$ is a one-sided Jacobi limit-periodic matrix with the spectrum on Julia $(T)$, moreover, its spectral measure $\sigma_{+}($supported on Julia $(T))$ possesses the renormalization property

$$
\int \frac{T^{\prime}(z) / d}{T(z)-x} d \sigma_{+}(x)=\int \frac{1}{z-x} d \sigma_{+}(x) .
$$

This means that $\sigma_{+}$is an eigen-measure for the Ruelle operator $L^{*} \sigma_{+}=\sigma_{+}$, where the operator $L$ acts on a continuous function $f$ on $\operatorname{Julia}(T)$ by

$$
(L f)(x)=\frac{1}{d} \sum_{T(y)=x} f(y) .
$$

In other words $\sigma_{+}$is the balanced measure on $\operatorname{Julia}(T)$.

Note that due to the Renormalization Equation the spectral measure of $J_{-}$ is the eigen-measure for the Ruelle operator

$$
\left(L_{2} f\right)(x)=\sum_{T(y)=x} \frac{f(y)}{T^{\prime}(y)^{2}}
$$

i.e. $L_{2}^{*} \sigma_{-}=\rho \sigma_{-}, \rho>0$. In the case of quadratic polynomials this fact was proved in [8]. 


\section{Appendix}

Here we recall some basic facts on two-sided Jacobi matrices. Let $J$ define a bounded selfadjoint operator on $l^{2}(\mathbb{Z})$. The resolvent matrix-function is defined by the relation

$$
W(z)=W(z, J)=\left[\begin{array}{ll}
\left\langle 0\left|(J-z)^{-1}\right| 0\right\rangle & \left\langle 0\left|(J-z)^{-1}\right| 1\right\rangle \\
\left\langle 1\left|(J-z)^{-1}\right| 0\right\rangle & \left\langle 1\left|(J-z)^{-1}\right| 1\right\rangle
\end{array}\right] .
$$

This matrix-function has an integral representation

$$
W(z)=\int \frac{d \sigma(x)}{x-z}
$$

with $2 \times 2$ matrix-measure having a compact support on $\mathbb{R}$. $J$ is unitary equivalent to the multiplication operator by an independent variable on

$$
L_{\sigma}^{2}=\left\{f=\left[\begin{array}{l}
f_{0}(x) \\
f_{1}(x)
\end{array}\right]: \int f^{*} d \sigma f<\infty\right\},
$$

moreover, under this unitary mapping from $l^{2} \rightarrow L_{\sigma}^{2}$ we have

$$
|0\rangle \mapsto\left[\begin{array}{l}
1 \\
0
\end{array}\right], \quad|1\rangle \mapsto\left[\begin{array}{l}
0 \\
1
\end{array}\right]
$$

Let $r_{-}(z)=r_{-}(z, 0), r_{+}(z)=r_{+}(z, 1)$ be resolvent functions of $J_{-}=J_{-}(0)$ and $J_{+}=J_{+}(1)$ respectively (see $(2.2)$ ), and $\sigma_{ \pm}$be the corresponding (scalar!) spectral measures. Then

$$
W(z)=\left[\begin{array}{cc}
r_{-}^{-1}(z) & p_{1} \\
p_{1} & r_{+}^{-1}(z)
\end{array}\right]^{-1} .
$$

Recall that according to our notation $p_{1} Q_{d}$ is the orthonormal polynomial of the degree $d-1$ for the measure $\sigma_{+}$and $p_{1} R_{d}$ is the related polynomial of the second kind:

$$
p_{1} R_{d}(z)=\int d \sigma_{+}(x) \frac{p_{1} Q_{d}(x)-p_{1} Q_{d}(z)}{x-z} .
$$

In this notations

$$
|d\rangle \mapsto \mathcal{E}_{d}(x):=\left[\begin{array}{c}
-p_{1}^{2} R_{d}(x) \\
p_{1} Q_{d}(x)
\end{array}\right]
$$

moreover

$$
\left[\begin{array}{c}
0 \\
p_{1} R_{d}(x)
\end{array}\right]=\int d \sigma(x) \frac{\mathcal{E}_{d}(x)-\mathcal{E}_{d}(z)}{x-z}
$$

Lemma 5.1. Let

$$
\mathcal{F}(x):=\left[\begin{array}{cc}
1 & -p_{1}^{2} R_{d}(x) \\
0 & p_{1} Q_{d}(x)
\end{array}\right], \quad \mathcal{G}(x):=\left[\begin{array}{cc}
0 & 0 \\
0 & p_{1} R_{d}(x)
\end{array}\right],
$$

Then

$$
\left[\begin{array}{ll}
\left\langle 0\left|(J-z)^{-1}\right| 0\right\rangle & \left\langle 0\left|(J-z)^{-1}\right| d\right\rangle \\
\left\langle d\left|(J-z)^{-1}\right| 0\right\rangle & \left\langle d\left|(J-z)^{-1}\right| d\right\rangle
\end{array}\right]=\mathcal{F}^{*}(\bar{z}) W(z) \mathcal{F}(z)+\mathcal{F}^{*}(\bar{z}) \mathcal{G}(z)
$$


Proof. This is a standard trick from the theory of orthogonal polynomials. Due to the unitary mapping onto $L_{\sigma}^{2}$, equivalently we have to calculate

$$
\int \frac{\mathcal{F}(x)^{*} d \sigma(x) \mathcal{F}(x)}{x-z} .
$$

Therefore, using orthogonality and (5.8), we continue

$$
\begin{aligned}
& =\int \frac{\mathcal{F}(x)^{*}-\mathcal{F}(\bar{z})^{*}}{x-z} d \sigma(x) \mathcal{F}(x)+\mathcal{F}(\bar{z})^{*} \int \frac{d \sigma(x) \mathcal{F}(x)}{x-z} \\
& =\mathcal{F}(\bar{z})^{*} \int d \sigma(x) \frac{\mathcal{F}(x)-\mathcal{F}(z)}{x-z}+\mathcal{F}(\bar{z})^{*} \int \frac{d \sigma(x)}{x-z} \mathcal{F}(z) \\
& =\mathcal{F}^{*}(z) \mathcal{G}(z)+\mathcal{F}^{*}(\bar{z}) W(z) \mathcal{F}(z) .
\end{aligned}
$$

Corollary 5.2. Combining (5.5) with (5.10) we get (2.23) from

$$
\begin{aligned}
& {\left[\begin{array}{ll}
\left\langle 0\left|(J-z)^{-1}\right| 0\right\rangle & \left\langle 0\left|(J-z)^{-1}\right| d\right\rangle \\
\left\langle d\left|(J-z)^{-1}\right| 0\right\rangle & \left\langle d\left|(J-z)^{-1}\right| d\right\rangle
\end{array}\right] } \\
= & \frac{\left[\begin{array}{ll}
\left\langle 0\left|(\tilde{J}-T(z))^{-1}\right| 0\right\rangle & \left\langle 0\left|(\tilde{J}-T(z))^{-1}\right| 1\right\rangle \\
\left\langle 1\left|(\tilde{J}-T(z))^{-1}\right| 0\right\rangle & \left\langle 1\left|(\tilde{J}-T(z))^{-1}\right| 1\right\rangle
\end{array}\right]}{T^{\prime}(z) / d},
\end{aligned}
$$

which is a part of the Renormalization Equation.

Proof. A straightforward computation.

\section{References}

[1] J. Avron and B. Simon, Singular continuous spectrum for a class of almost periodic Jacobi matrices Bull. Amer. Math. Soc. (N.S.) 6 (1982) 81-85.

[2] M. F. Barnsley, J. S. Geronimo, and A. N. Harrington, Almost periodic Jacobi matrices associated with Julia sets for polynomials, Comm. Math. Phys. 99 (1985), no. 3, 303-317.

[3] J. Bellissard, D. Bessis, and P. Moussa, Chaotic states of almost periodic Schrödinger operators, Phys. Rev. Lett. 49 (1982), no. 10, 701-704.

[4] J. Bellissard and B. Simon, Cantor spectrum for almost Mathieu equation J. Funct. Anal. 48 (1982), no. 3, 408-419.

[5] R. Bowen, Equilibrium States and the Ergodic Theory of Anosov Diffeomorphisms Lecture Notes in Mathematics, v. 470, Springer-Verlag, Berlin-Heidelberg-New York, 1975.

[6] J. Herndon, Limit periodicity of sequences defined by certain recurrence relations; and Julia sets, Ph.D. thesis, 1985.

[7] O. Knill, Isospectral deformations of random Jacobi operators. Comm. Math. Phys. 151 (1993), no. 2, 403-426.

[8] M. Sodin and P. Yuditski, The limit-periodic finite-difference operator on $l_{2}(\mathbb{Z})$ associated with iterations of quadratic polynomials. J. Statist. Phys. 60 (1982), no. 5-6, 863-873.

[9] F. Pehersorfer, A. Volberg, and P. Yuditskii, Two weight Hilbert transform and Lipschitz property of Jacobi matrices associated to hyperbolic polynomials, submitted.

[10] , Limit periodic Jacobi matrices with a singular continuous spectrum and the renormalization of periodic matrices, arXiv: math-ph/0510019, pp. 1-25. 
Department of Mathematics, Michigan State University, East Lansing, Michigan 48824

E-mail address: volberg@math.msu.edu

Abteilung für Dynamische Systeme, und Approximationstheorie, Institut für Analysis, J. KePler Universität Linz, Linz, Austria A-4040

E-mail address: Franz.Peherstorfer@jku.at

Abteilung für Dynamische Systeme, und Approximationstheorie, Institut für Analysis, J. Kepler Universität Linz, Linz, Austria A-4040, and Department of Mathematics, Bar Ilan University, Ramat-Gan 52900, Israel

E-mail address: yuditski@macs.biu.ac.il 